ORIENTAL JOURNAL OF CHEMISTRY

An International Open Access, Peer Reviewed Research Journal

\title{
Synthesis, Characterization of Copper Complexes of 9H-Carbazole-3-carbaldehyde-4-phenylthiosemicarbazone, 10-Hexyl-10-H-phenothiazine-3-carbaldehyde-4-phenylthio semicarbazone and 2-Thiophenecarboxaldehyde-4-methyl- thiosemicarbazone and Anti-bacterial Activity Studies of Ligands and Complexes
}

\section{N. RAMA JYOTHI ${ }^{2 *}$, R. JAYA MADHURI ${ }^{3}$, N.A. MOHAMED FAROOK ${ }^{1}$ and K. GOWTHAMI ${ }^{3}$}

'Department of Chemistry, Khadir Mohideen College, Adhirampattinam, Tamil Nadu- 614 701, India. ${ }^{2}$ Department of Chemistry, School of Engineering, Sri Padmavathi Mahila Visvavidyalayam, Tirupati, Andhra Pradesh-517 501, India.

3 Department of Microbiology, Sri Padmavathi Mahila Visvavidyalayam, Tirupati, Andhra Pradesh - 517 501, India.

${ }^{*}$ Corresponding author E-mail: ramadasaradhi@gmail.com

http://dx.doi.org/10.13005/ojc/360615

(Received: October 09, 2020; Accepted: November 11, 2020)

ABSTRACT

In Coordination chemistry metal chelating agents has a vital role, among them thiosemicarbazones occupies an important place due to their a range of applications in different fields, such as analytical and biological. In the literature we can find many thiosemicarbazones which has a wide range of applications both in pharmacy and chemical fields. But still there is a scope is there to synthesize new thiosemicarbazone ligands and their metal complexes due to their utility is still there in modern chemistry also. In this present study we synthesized the copper(II) complexes of 9H-Carbazole-3-carbaldehyde-4-phenylthiosemicarbazone, 10-hexyl-10$\mathrm{H}$-phenothiazine-3-carbaldehyde-4-phenylthiosemicarbazone and 2-thiophenecarboxalde-hyde4-methylthiosemicarbazone and these complexes are characterized with FT-IR, XRD analysis and thermal stabilities of the both ligands and complexes are compared with thermogravimetric analysis studies. Finally the antibacterial activities of both chelating agents and their metal complexes are tested with two Gram-positive bacterial stains, such as Bacillus subtilis, Staphylococcus aureus and two Gram-negative bacterial stains, Pseudomonas aeruginosa, Escherichia coli.

Keywords: 9H-Carbazole-3-carbaldehyde-4-phenylthiosemi carbazone, 10-Hexyl-10-Hphenothiazine-3-carbaldehyde-4-phenyl thiosemicarbazone, 2-Thiophenecarboxaldehyde-4methylthiosemicarbazone, copper(II) complexes, antibacterial studies.

This is an Open Access article licensed under a Creative Commons license: Attribution 4.0 International (CC- BY). Published by Oriental Scientific Publishing Company @ 2018 


\section{INTRODUCTION}

Organic chelating agents plays an important role in both analytical and biological fields. Among organic reagents, thiosemicarbazones have a vital role due to the ability of formation of bonds with enzymes in biological systems. The donor atoms of nitrogen and sulphur of thiosemicarbazones forms a chelating bonds with the wide range of metal ions $\mathrm{s}^{1-4}$. In recent years, so many authors were reported regarding the synthesis and characterization studies of thiosemicarbazones and their metal complexes. But still its potentiality has not been exposed yet. Its owes us to synthesize new thiosemicarbazone metal complexes and to study their biological activities. Metal complexes of thiosemicarbazones were proven as anti-bacterial $\left.\right|^{5,6,7}$, anti-fungal $\left.\right|^{8,9}$, anti-cancer ${ }^{10,11}$ agents. Chandra and Vandana ${ }^{12}$ had reported the anti-bacterial and anti-cancer activities of copper and nickel complexes of 2-carboxybenzaldehyde thiosemicarbazone. Khan et al., ${ }^{13}$ reported about the synthesis, characterization and antibacterial properties of thiosemicarbazone ligand and their copper, nickel, and cobalt complexes. Piri et al.,$^{14}$ reported the anti-oxidant and anti-bacterial activities of $\mathrm{Cu}(\mathrm{II})$ complexes of thiosemicarbazone ligands.

A recent study ${ }^{15}$ reported the antibacterial activity of cinnamaldehyde thiosemicarbazones against the bacterial stains, such as E. coli and K. pneumoniae. In this study they tested the antibacterial activity of thiosemicarbazone ligands and their $\mathrm{Cu}(\mathrm{II})$ and $\mathrm{Zn}(\mathrm{II})$ complexes. This study found that the compound which has lowest partition co-efficient has more antibacterial activities on the tested bacteria stains. Based on the results they concluded that the metalloantibiotics are a reliable compounds in fighting against the bacterial stains. Copper, cobalt and nickel metal complexes of coumarine-3-yl thiosemicarbazone compounds were tested for antibacterial properties towards few Gram +ve and Gram-ve bacterial stains by Refat et al., ${ }^{16}$. Naveen et al., ${ }^{17}$ reported about the study related to substituted triazole thiosemicarbazone derivatives preparation and their biological activities. In this study they found that few of their tested compounds were had more potential activity against $B$. subtilis and $P$. aeruginosa than a standard reference drug, Ciprofloxacin. The antibacterial studies performed in this study was supported by molecular docking studies also.
Kumar et al., ${ }^{18}$ reported the synthesis and antibacterial activities of $\mathrm{Co}(\mathrm{II}), \mathrm{Cu}(\mathrm{II}), \mathrm{Ni}(\mathrm{II})$, and $\mathrm{Zn}$ (II) complexes of acenaphthoquinone 3-(4-benzylpiperidyl) thiosemicarbazones against Escherichia coli, Pseudomonas aeruginosa and $S$. aureus and $B$. subtilis. In this study they reported $\mathrm{Ni}$ (II) complexes had greater activity than other three metal complexes against the tested bacterial stains. Antibacterial and anti-tumor activities of diorganotin complexes with 3-methoxy salicylaldehyde thiosemicarbazone was reported by Khandani et al., ${ }^{19}$. They tested the chelating agents and their metal complexes towards the bacteria stains, reported that one of the metal complex shows comparative activity along with the standard drugs.

Akbari et al., ${ }^{20}$ studied the antibacterial activities of $\mathrm{Ni}$ (II) complexes of isothiosemi carbazone and thiosemicarbazone ligands. This study reports one of the $\mathrm{Ni}(\mathrm{II})$ complexes had higher antibacterial property against the Staphylococcus aureus than other complexes. The inhibition zone and minimal inhibition concentration of $\mathrm{Ni}(\mathrm{II})$ complex with higher activity was found that $30 \mathrm{~mm}$, and $1.56 \mathrm{mg} / \mathrm{mL}$, respectively.

The present study reports the synthesis and characterization of copper(II) complexes of $9 \mathrm{H}$-Carbazole-3-carbaldehyde4-phenylthiosemicarbazone (Cu-CCPTSC), 10-Hexyl-10-H-pheno thiazine-3-carbaldehyde -4-phenylthiosemicarbazone (CUHPCPTSC), 2-Thiophenecarboxalde -hyde-4-methylthiosemicarbazone (Cu-TCMTSC) with FT-IR, XRD and thermogravimetric analysis. Antibacterial activities of both chelating agents and their copper complexes against two Gram positive bacterial stains, Bacillus subtilis, Staphylococcus aureus and two Gram negative bacterial stains, Pseudomonas aeruginosa, Escherichia coli.

\section{EXPERIMENTAL}

\section{Synthesis of ligands}

The ligands which are used for the chelation with copper metal ions, such as $9 \mathrm{H}$-carbazole3-carbaldehyde-4-phenyl-3-thiosemicarbazone (CCPTSC), 10-Hexyl-10-H-phenothiazine-3carb -aldehyde-4-phenylthiosemicarbazone (HPCPTSC) and 2-Thiophenecarboxaldehyde4-methyl thiosemi-carbazone (TCMTSC) were synthesized as per literature ${ }^{21,22}$. 
Instrumentation

Infrared spectrum

The FT-IR spectra were recorded on a Nicolet FT-IR 560 Magna spectrometer.

\section{XRD spectra}

Powder XRD (PAN analytical X'Pert PRO, USA) was carried out using CuK $\alpha$ (0.154056 nm) radiation at $40 \mathrm{kV}$ and $30 \mathrm{~mA}$. The data was collected between 10 and $70^{\circ} 2 \theta$ with a step size of $0.02^{\circ}$.

\section{Thermogravimetric analysis}

Thermogravimetric analysis was carried out in the temperature range of $25-800^{\circ} \mathrm{C}$ in nitrogen atmosphere and a heating range of $10^{\circ} \mathrm{C} / \mathrm{min}$ using SDT Q600 V20.9 Build 20 (TA instruments, Waters, USA).

Preparation of copper(II) complex of $9 \mathrm{H}$-carbazole3-carbaldehyde-4-phenyl-3-thio semi carbazone (Cu-CCPTSC)

Methanolic solutions of $0.34 \mathrm{~g}$ of $9 \mathrm{H}$ carbazole-3-carbaldehyde-4-phenyl-3-thiosemicarba -zone in $100 \mathrm{~mL}$ and $0.09 \mathrm{~g}$ of copper(II) chloride dihydrate in $100 \mathrm{~mL}$ were refluxed about 4 hours. in a flask. Obtained grass green product (with yield, $86 \%$ ) which is then separated through the filtration. This filtered product was then dried. To obtain the pure product it was then recrystallized with methanol. A possible structure of copper(II) complex of $9 \mathrm{H}$-carbazole-3-carbaldehyde-4-phenyl3-thiosemicarbazone is presented in Scheme 1.<smiles>FC(F)(Cl)C(F)(F)C(F)(F)C(Cl)(Cl)Cl</smiles>
$\mathrm{Cl}-$

Scheme 1. Possible structure of copper(II) complex of $9 \mathrm{H}-$ carbazole-3-carbaldehyde-4-phenyl-3-thiosemicarbazone

Preparation of copper(II) complex of HPCPTSC Methanolic solutions of $0.23 \mathrm{~g}$ of HPCPTSC in $100 \mathrm{~mL}$ and $0.045 \mathrm{~g}$ of copper(II) chloride dihydrate in $100 \mathrm{~mL}$ were refluxed for nearly $4 \mathrm{~h}$. in a RB flask. Obtained grey product (with an yield, 89\%) was separated through the filtration and dried over. Final product then recrystallizes from methanol. A possible structure of copper(II) complex of HPCPTSC is presented in Scheme 2.<smiles>CCCCCCN(/N=C/c1ccc2c(c1)sc1ccccc1n(CCCCCC)n2CCCCC)C(=S)NC(=S)Nc1ccccc1</smiles>

Scheme 2. Possible structure of copper(II) complex of HPCPTSC

Preparation of copper(II) complex of 2-thiophenecarboxaldehyde-4-methyl-3-thiosemicarba zone (Cu-TCMTSC)

Copper(II) complex of 2-thiophenecarboxaldehyde-4-methyl-3-thiosemicarbazone has synthesizes by heating an $50 \mathrm{~mL}$ methanolic 2-thiophene carboxaldehyde-4-methyl-3-thiosemi carbazone ( $0.40 \mathrm{~g})$ and $0.18 \mathrm{~g}$ of copper(II) chloride dihydrate in $50 \mathrm{~mL}$ of methanol for nearly 4 and half an hour in a RB flask. A persistent blue product was formed (with an yield, $81 \%$ ) has took off through filtration, which was then dried. Then final product recrystallizes with methanol. A possible structure of copper(II) complex of 2-thiophene carboxaldehyde-4-methyl-3-thiosemicarbazone is presented in Scheme 3.

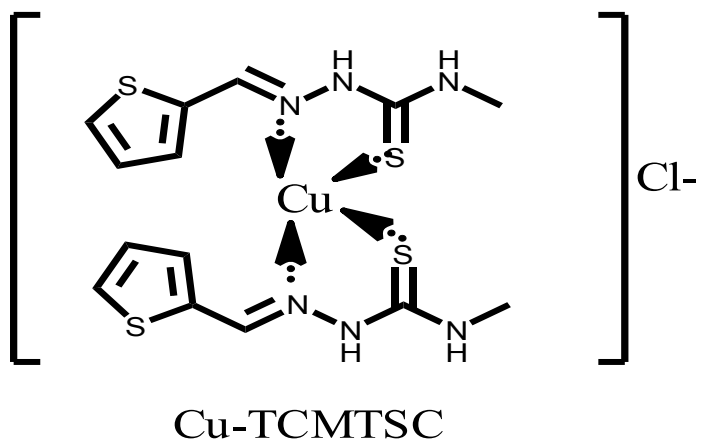

Scheme 3. Possible structure of copper(II) complex of 2-thiophenecarboxaldehyde-4-methyl-3-

Experimental procedure for anti-bacterial activity

Antibacterial properties of the ligands (1-9) and their complexes (1A-9A) screened towards two Gram +ve bacterial stains, B. subtilis, $S$. aureus and two Gram-ve bacterial stains $P$. aeruginosa, E. coli using agar well diffusion method ${ }^{26}$. Stock solution was prepared by dissolving $1 \mathrm{mg}$ of the test 
compounds in $10 \mathrm{~mL}$ of dimethyl sulphoxide and further diluted to prepare $100 \mu \mathrm{g} / \mathrm{mL}$ concentrations. The antibiotic Tetracycline was used as standard for comparing activity of the tested compounds. Freshly prepared Muller Hinton Agar medium $(20 \mathrm{~mL})$ was poured in each Petri plate. After solidification of the medium, $24 \mathrm{~h}$ cultures were prepared for microorganisms B. subtilis, S. aureus, P. aeruginosa and E. coli containing approximately 105-106 colony forming units (CFU) per $\mathrm{mL}$ was spread on the surface of the medium. Sterile swabs are used to swab in the culture on nutrient agar plate for making microbial lawn on sterile nutrient agar plate. Sterile filter papers were placed on laun. The different concentration of compounds are 25,50,75 and 100 $\mu \mathrm{L}$ and positive and negative control were placed on the disc. After $24 \mathrm{~h}$ inhibition of the test pathogens by the synthesized compounds around the wells was measured to determine the antibacterial (zone of inhibition) activity of the samples were studied to know the potency. The experiments were conducted in triplicate and average tabulated as final result.

\section{RESULT AND DISCUSSION}

\section{Characterization of Cu-CCPTSC, Cu-HPCPTSC and Cu-TCMTSC}

Copper(II) complexes of CCPTSC, HPCPTSC and TCMTSC are characterized with FTIR, XRD. Thermogravimetric studies were performed to compare the thermal stabilities of ligands and their metal complexes.

\section{FT-IR analysis of Cu-CCPTSC, Cu-HPCPTSC and Cu-TCMTSC}

FT-IR spectra of free ligands CCPTSC, HPCPTSC and TCMTSC are presented in our previous studies ${ }^{21,22}$. By comparing the spectra of complexes with free ligands it is confirmed the chelation of metal ion with free ligands.

FT-IR spectrum of complex Cu-CCPTSC is presented in Fig. 1. The IR spectra of complex $4 \mathrm{~A}$ is represented as: Carbon-Nitrogen peak appears at $1581 \mathrm{~cm}^{-1}$ and Carbon-Sulphur peak at $1151 \mathrm{~cm}^{-1}$. When this data compared with its parent ligand, Carbon-Nitrogen peak is changed from 1635 to 1581 $\mathrm{cm}^{-1}$. Carbon-Sulphur peak is changed from 1222 to $1151 \mathrm{~cm}^{-1}$, this change is due to complexation of metal with chelating agent. This shift confirms the formation of the complex CU-CCPTSC.
FT-IR spectrum of complex HPCPTSC is presented in Fig. 2. The spectral data of complex $5 \mathrm{~A}$ is represented as Carbon-Nitrogen peak appears at $1558 \mathrm{~cm}^{-1}$ and Carbon-Sulphur peak appears at $1148 \mathrm{~cm}^{-1}$. When this data compared with its parent ligand Carbon-Nitrogen peak is changed from 1625 to $1558 \mathrm{~cm}^{-1}$ and CarbonSulphur peak is changed from 1236 to $1148 \mathrm{~cm}^{-1}$, this change is due to complexation of metal with chelating agent. This data indicates the formation of complex Cu- HPCPTSC.

In Fig. 3 IR spectrum of complex CuTCMTSC is presented. IR spectra of complex CuTCMTSC is presented as, Carbon-Nitrogen peak appears at $1581 \mathrm{~cm}^{-1}$ and Carbon-Sulphur peak appears at $1173 \mathrm{~cm}^{-1}$. When this data compared with its parent ligand, $\mathrm{C}=\mathrm{N}$ peak is shifted from 1624 to $1581 \mathrm{~cm}^{-1}$ and $\mathrm{C}=\mathrm{S}$ peak is shifted from 1232 to $1173 \mathrm{~cm}^{-1}$, this shift is due to the chelation of metal ion to the chelating agent. This shift confirms the formation of the complex CU-TCMTSC.

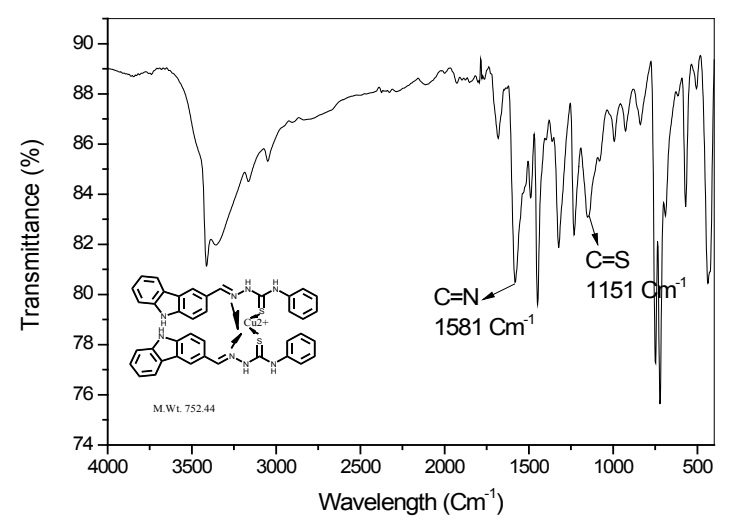

Fig. 1. FT-IR Spectrum of complex Cu-CCPTSC

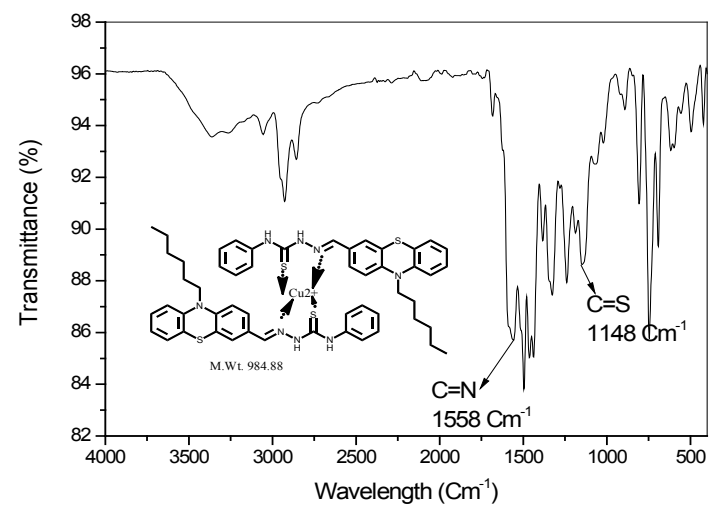

Fig. 2. IR Spectrum of complex Cu- HPCPTSC 


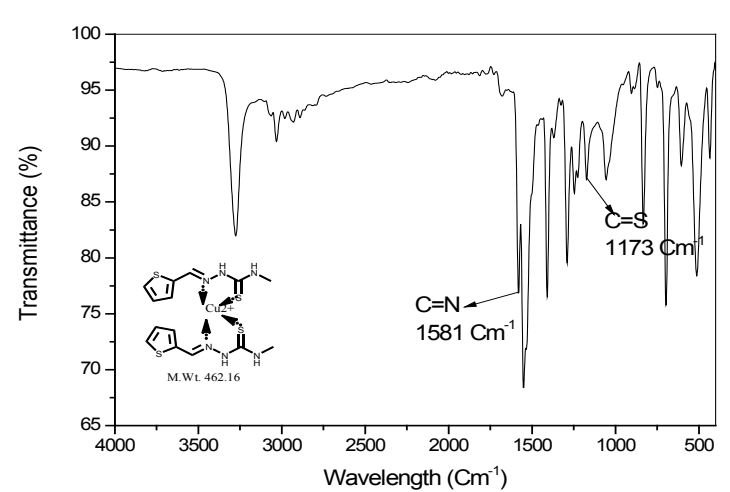

Fig. 3. FT-IR Spectrum of complex Cu-TCMTSC

XRD analysis of Cu-CCPTSC, Cu-HPCPTSC and CU-TCMTSC

XRD studies were performed to identify crystalline nature of copper(II) complexes of thiosemicarbazone ligands. Fig. 4 to 6 represents the XRD spectra of the complexes CU-CCPTSC, Cu-HPCPTSC and CU-TCMTSC, respectively. From the diffractograms it is observed that the dominant $2 \theta$ XRD peaks for the complexes Cu-CCPTSC, Cu-HPCPTSC and Cu-TCMTSC are appears at $22.30^{\circ}, 16.19^{\circ}$ and 6.33, respectively. Except $\mathrm{Cu}-$ CCPTSC, in all the remaining complexes the dominant $2 \theta$ XRD peaks are shifted to lower side than their parent ligands. The morphology of ligands has completely changed due to the chelation with copper(II) ion.

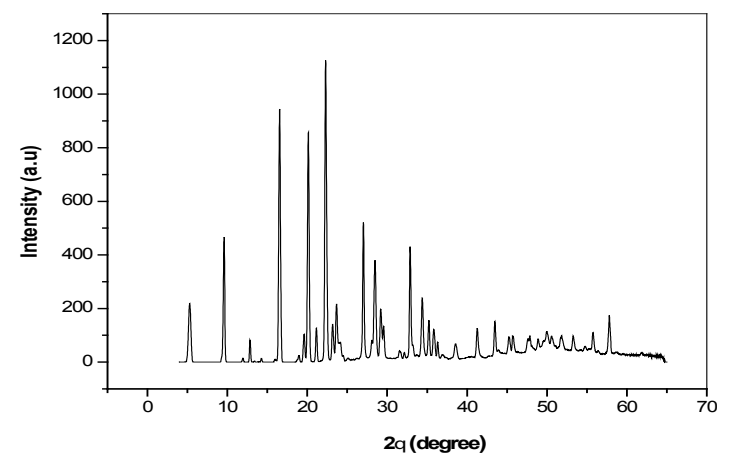

Fig. 4. XRD diffractogram of complex Cu-CCPTSC

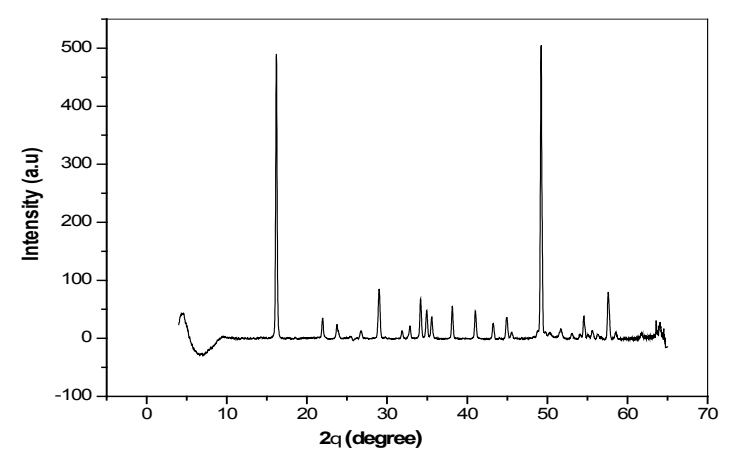

Fig.5. XRD diffractogram of complex Cu-HPCPTSC

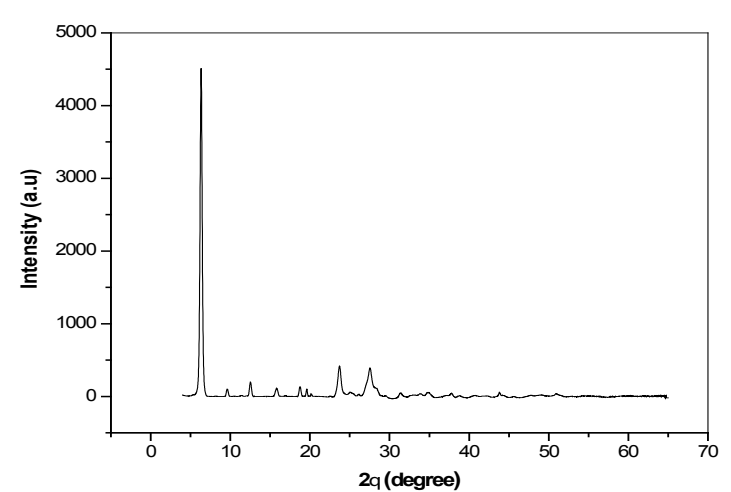

Fig.6. XRD diffractogram of complex Cu-TCMTSC

Thermogravimetric analysis of Cu-CCPTSC, Cu-HPCPTSC and Cu-TCMTSC

The thermogravimetric curve of complex Cu-CCPTSC is presented in Fig. 7. The thermogravimetric curve of complex Cu-CCPTSC shows that the complex starts melting at $100^{\circ} \mathrm{C}$, the decomposition observed at $250^{\circ} \mathrm{C}$ and $525^{\circ} \mathrm{C}$ with a weight loss of $36 \%$ and $56 \%$, respectively and almost $63.5 \%$ weight loss observed at $800^{\circ} \mathrm{C}$ by remaining $36.5 \%$ of the complex. The thermogravimetric curve of complex Cu-HPCPTSC is presented in Fig. 8. From the thermogravimetric curve of complex Cu-HPCPTSC, it is observed that the complex starts melting at $260^{\circ} \mathrm{C}$ and the decomposition observed at $490^{\circ} \mathrm{C}$ with a weight loss of $60 \%$ and more than $65 \%$ weight loss observed at $800^{\circ} \mathrm{C}$. The thermogravimetric curve of complex Cu-TCMTSC indicates that the compound starts melting at $210^{\circ} \mathrm{C}$ and the decomposition observed at $325^{\circ} \mathrm{C}$ with a weight loss $58 \%$ and more than $77 \%$ weight loss observed at $800^{\circ} \mathrm{C}$. Thermogravimetric curve of complex Cu-TCMTSC is presented Fig. 9. The thermal stability of the complexes is follows the order CU-CCPTSC > Cu-HPCPTSC > Cu-TCMTSC.

When compare the thermal stabilities of thiosemicarbazone ligands (1-9) and their copper(II) complexes (1A-9A) the following points are predicted.

The complex CU-CCPTSC remained at $800^{\circ} \mathrm{C}$ is $31 \%$ more than its parent ligand at the same temperature. The thermal stability of the ligand is increases with the chelation of metal ion. This is may be attributed to the formation of complex which has the increase in molecular weight and also the formation of metal to ligand bond also takes part in the increase in the thermal stability of the complex. 
The complex Cu-HPCPTSC remained at $800^{\circ} \mathrm{C}$ is $33 \%$ more than its parent ligand at the same temperature. The complex Cu-TCMTSC remained at $800^{\circ} \mathrm{C}$ is $21 \%$ more than its parent ligand at the same temperature.

From the above, it is concluded that all the complexes are having greater thermal stabilities than their parent ligands. Among the complexes, which are formed from phenylthiosemicarba zone ligands are having greater stability than the complex formed from methylthiosemicarbazone ligand. Both the complexes formed from phenylthiosemicarbazone ligands are remained nearly $40 \%$ at $800^{\circ} \mathrm{C}$. But the complex formed from methylthiosemicarbazone ligand is left only $20 \%$ after $800^{\circ} \mathrm{C}$. Based on this it is assumed that phenyl group presence also takes part in the increase in the thermal stability of the compounds. The presence of the metal ion also one of the reasons for the increase in the thermal stability of the ligand. The stronger the metal to ligand bond may also enhances the thermal stability of the ligand. Copper metal ions shows greater affinity for thiosemicarbazone ligands due to the presence of sulphur atom.

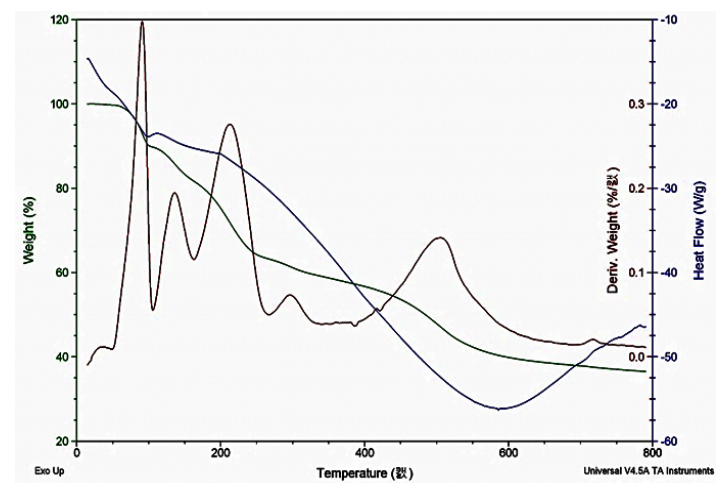

Fig. 7. TGA curve of complex Cu-CCPTSC

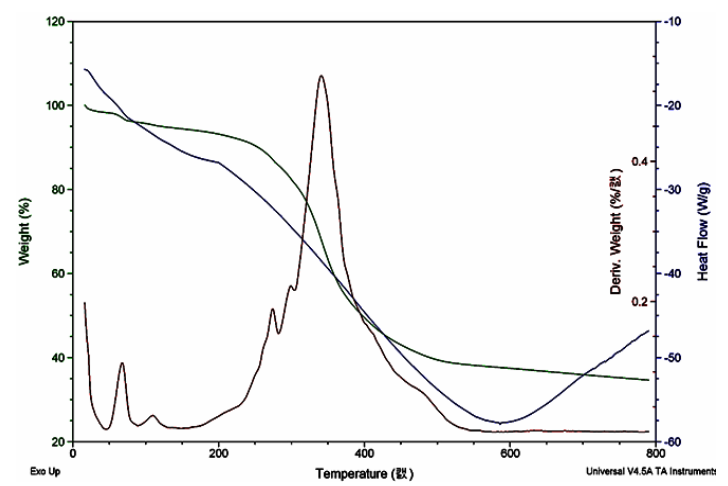

Fig. 8. TGA curve of complex Cu-HPCPTSC

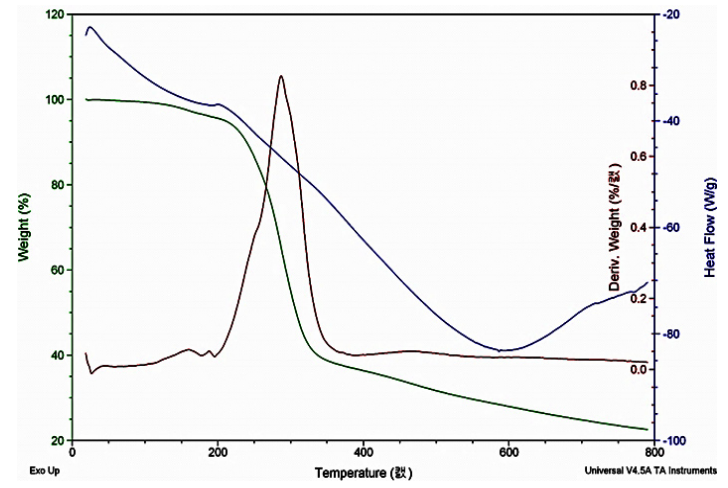

Fig. 9. TGA curve of complex Cu-TCMTSC

Antibacterial studies of chelating agents and their copper complexes

To evaluate antibacterial properties of newly synthesized nine chelating agents and their complexes with copper towards two Gram+ve bacteria, B. subtilis, S. aureus and two Gram-ve bacteria, P. aeruginosa, E.coli. For this purpose four different concentrations ( $25,50,75$ and $100 \mu \mathrm{L}$ ) of chelating agents and their copper complexes were used. The data obtained in this study is presented in Table 1. Based on the data obtained in this study, the order of reactivity of nine chelating agents and their copper complexes is presented in Table 2.

The antibacterial activity of parent ligand is increases with all tested four bacterial stains. In case of $B$. subtilis there is a very slight increase in activity is observed in ligand 4 by chelation with copper(II). In case of E.coli, there is a huge change in activity is observed in ligand 4 by chelation with metal ion (Table 2). In this case almost the activity is doubled by chelation with metal ion. In case of $S$. aureus and $P$. aeruginosa there is a uniformity of increase in activity of ligand 4 and $4 \mathrm{~A}$ at all concentrations. The complex $5 \mathrm{~A}$ has higher activity than its parent ligand 5 among all tested bacterial stains. The increase in activity is very marginal in case of $B$. subtilis and $P$. aeruginosa at all concentrations. But significant increase in activity is observed in $S$. aureus. High increase in activity is observed in case of $E$. coli due to chelation with copper(II). In case of $B$. subtilis and $P$. aeruginosa there is a slight increase in complex $6 \mathrm{~A}$ compare to its parent ligand 6 is observed at all concentrations. In case of $S$. aureus, at low concentration $(25 \mu \mathrm{L}) 6 \mathrm{~A}$ exhibits nearly 3 times activity than its parent ligand but high concentration $(100 \mu \mathrm{L})$ only $50 \%$ increase in activity is observed. 
In case of E.coli, at all concentrations nearly 2 times higher activity is observed for $6 \mathrm{~A}$ than its ligand 6 . Among the ligands and complexes, ligands exhibit some inhibitory biological activity where as complexes at higher concentration shows best activity. Minimal inhibition zones (in $\mathrm{mm}$ ) values gives an interesting information about bacterial action towards the compound. From these studies, it is proven that metal thiosemicarbazones are having potential antibacterial activity. The reason for the activity of the metal thiosemicarbazones can be established based on structure-activity relationship studies.

Table 1: Minimal inhibition zones (in $\mathrm{mm}$ ) of ligands (and their copper(II) complexes against four bacterial stains at four different concentrations

\begin{tabular}{ccccccccccccccccc}
\hline \multirow{2}{*}{ Concentration( $\mu \mathrm{L})$} & \multicolumn{3}{c}{ B. subtilis } & \multicolumn{4}{c}{ S. aureus } & \multicolumn{4}{c}{ P. aeruginosa } \\
& 25 & 50 & 75 & 100 & 25 & 50 & 75 & 100 & 25 & 50 & 75 & 100 & 25 & 50 & 75 & 100 \\
\hline CCPTSC & 8.0 & 18.0 & 21.0 & 25.0 & 6.0 & 12.0 & 15.0 & 19.0 & 8.0 & 15.0 & 17.0 & 21.0 & 2.0 & 5.0 & 7.0 & 9.0 \\
Cu-CCPTSC & 14.0 & 19.0 & 20.0 & 28.0 & 10.0 & 22.0 & 26.0 & 30.0 & 14.0 & 22.0 & 25.0 & 31.0 & 8.0 & 13.0 & 19.0 & 21.0 \\
HPCPTSC & 7.0 & 13.0 & 18.0 & 25.0 & 8.0 & 14.0 & 18.0 & 22.0 & 3.0 & 10.0 & 12.0 & 24.0 & 4.5 & 9.0 & 13.0 & 16.0 \\
Cu-HPCPTSC & 11.0 & 14.0 & 20.0 & 26.0 & 18.0 & 20.0 & 25.0 & 31.0 & 5.0 & 12.0 & 14.0 & 25.0 & 10.0 & 19.0 & 22.0 & 24.0 \\
TCMPTSC & 4.0 & 9.0 & 11.0 & 17.0 & 6.0 & 10.0 & 14.0 & 17.0 & 5.0 & 10.0 & 14.0 & 21.0 & 5.0 & 9.0 & 10.0 & 12.0 \\
Cu-TCMTSC & 10.0 & 12.0 & 14.0 & 21.0 & 18.0 & 20.0 & 22.0 & 26.0 & 8.0 & 12.0 & 19.0 & 25.0 & 13.0 & 17.0 & 20.0 & 25.0 \\
\hline
\end{tabular}

Table 2: Order of reactivity of ligands and their copper(II) complexes against four bacterial stains at four different concentrations

\begin{tabular}{|c|c|c|c|}
\hline S.No. & Conc. $(\mu \mathrm{L})$ & Compounds & Order of reactivity \\
\hline \multirow[t]{8}{*}{1} & 25 & CCPTSC & B. subtilis $=P$. aeruginosa $>S$. aureus $>$ E.coli \\
\hline & & Cu-CCPTSC & B. subtilis $=P$. aeruginosa $>S$. aureus $>$ E. coli \\
\hline & 50 & CCPTSC & B. subtilis $>P$. aeruginosa $>S$. aureus $>$ E. coli \\
\hline & & Cu-CCPTSC & S. aureus $=P$. aeruginosa $>B$. subtilis $>$ E.coli \\
\hline & 75 & CCPTSC & B. subtilis $>P$. aeruginosa $>S$. aureus $>$ E.coli \\
\hline & & Cu-CCPTSC & S. aureus $>P$. aeruginosa $>B$. subtilis $>$ E.coli \\
\hline & 100 & CCPTSC & B. subtilis $>P$. aeruginosa $>S$. aureus $>$ E.coli \\
\hline & & Cu-CCPTSC & P. aeruginosa $>S$. aureus $>B$. subtilis $>$ E.coli \\
\hline \multirow[t]{8}{*}{2} & 25 & HPCPTSC & S. aureus $>B$. subtilis $>$ E.coli>P. aeruginosa \\
\hline & & Cu-HPCPTSC & S. aureus $>$ B. subtilis $>$ E.coli>P. aeruginosa \\
\hline & 50 & HPCPTSC & S. aureus $>B$. subtilis $>P$. aeruginosa $>$ E.coli \\
\hline & & Cu-HPCPTSC & S. aureus $>$ E.coli $>B$. subtilis $>P$. aeruginosa \\
\hline & 75 & HPCPTSC & B. subtilis $=S$. aureus $>$ E.coli $>P$. aeruginosa \\
\hline & & Cu-HPCPTSC & S. aureus $>$ E.coli $>$ B. subtilis $>$ P. aeruginosa \\
\hline & 100 & HPCPTSC & B. subtilis $>P$. aeruginosa $>S$. aureus $>$ E.coli \\
\hline & & Cu-HPCPTSC & S. aureus $>P$. aeruginosa $>B$. subtilis $=$ E.coli \\
\hline \multirow[t]{8}{*}{3} & 25 & TCMPTSC & S. aureus $>P$. aeruginosa $=E$.coli $>B$. subtilis \\
\hline & & Cu-TCMTSC & S. aureus $>$ E.coli $>B$. subtilis $>P$. aeruginosa \\
\hline & 50 & TCMPTSC & S. aureus $=P$. aeruginosa $>B$. subtilis $=E$. coli \\
\hline & & Cu-TCMTSC & S. aureus $>$ E. coli. aeruginosa $=B$. subtilis \\
\hline & 75 & TCMPTSC & S. aureus $=P$. aeruginosa $>B$. subtilis $>$ E.coli \\
\hline & & Cu-TCMTSC & S. aureus $>$ E.coli>P. aeruginosa $>B$. subtilis \\
\hline & 100 & TCMPTSC & $P$. aeruginosa $>B$. subtilis $=S$. aureus $>$ E.coli \\
\hline & & Cu-TCMTSC & S. aureus $>P$. aeruginosa $=E$.coli $>B$. subtilis \\
\hline
\end{tabular}

Antibacterial activity of $9 \mathrm{H}$-carbazole-3-carbaldehyde4-phenyl-3-thiosemicarbazone (CCPTSC) and its copper(II) complex (Cu-CCPTSC)

Antibacterial activity of $9 \mathrm{H}$-carbazole3-carbaldehyde-4-phenyl-3-thiosemicarbazone (CCPTSC) and its copper(II) complex (Cu-CCPTSC) is presented in Figure 10.

The above figure indicates that chelation with copper(II) the antibacterial activity of parent ligand is increases with all tested bacterial stains. In case of $B$. subtilis there is a very slight increase in activity is observed in ligand CCPTSC by chelation with copper (II). In case of E.coli, there is a huge change in activity is observed in ligand CCPTSC by chelation with metal ion. In this case almost the activity is doubled by chelation with metal ion. In case of $S$. aureus and $P$. aeruginosa there is a uniformity 
of increase in activity of ligand CCPTSC and CU-CCPTSC at all concentrations.

Antibacterial activity of HPCPTSC and its copper(II) complex (Cu-HPCPTSC)

Antibacterial activity of HPCPTSC and its copper(II) complex (Cu-HPCPTSC) is presented in Figure 11.

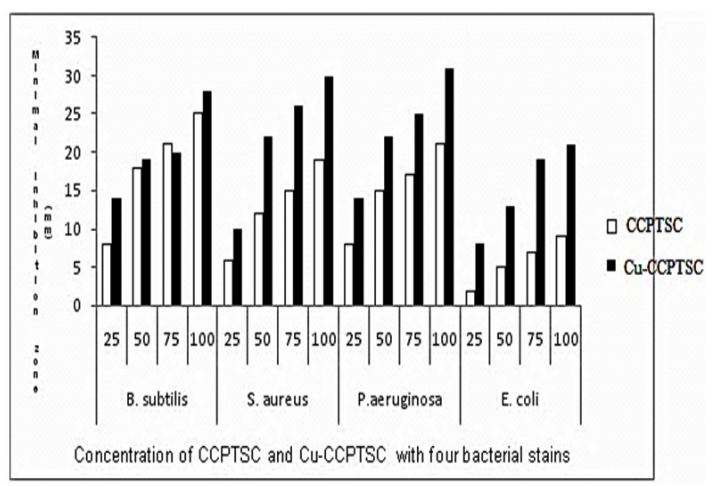

Fig. 10. Minimal inhibition zone $(\mathrm{mm})$ values of CCPTSC and Cu-CCPTSC against four bacterial stains

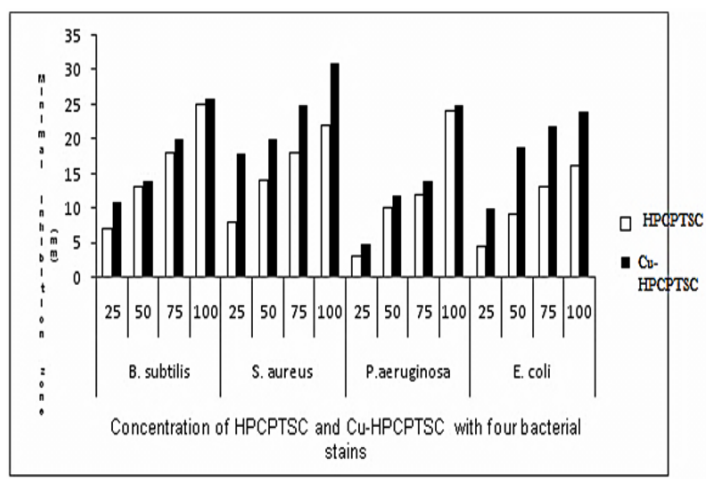

Fig. 11. Minimal inhibition zone values $(\mathrm{mm})$ of HPCPTSC and $\mathrm{Cu}$-HPCPTSC against four bacterial stains

The above figure indicates that the complex CU-HPCPTSC has higher activity than its parent ligand HPCPTSC among all tested bacterial stains. The increase in activity is very marginal in case of $B$. subtilis and $P$. aeruginosa at all concentrations. But significant increase in activity is observed in $S$. aureus. High increase in activity is observed in case of E.coli.

Antibacterial studies of 2-thiophenecarboxaldehyde-4-methyl-3-thiosemicarbazone (TCMTSC) and its copper(II) complex (Cu-TCMTSC)

Antibacterial study of 2-thiophenecarboxaldehyde-4-methyl-3-thiosemicarbazone (TCMTSC)and its copper(II) complex (CU-TCMTSC) is presented in Figure 12.

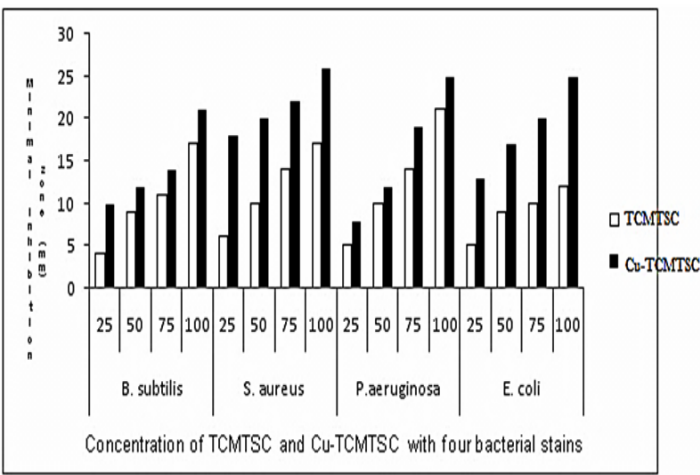

Fig. 12. Minimal inhibition zone values $(\mathrm{mm})$ of TCMTSC and $\mathrm{Cu}$-TCMTSC against four bacterial stains

The above figure indicates that chelation with copper(II) the antibacterial activity of parent ligand is increases with all tested bacterial stains. In case of $B$. subtilis and $P$. aeruginosa there is a slight increase in complex Cu-TCMTSC compare to its parent ligand TCMTSC is observed at all concentrations. In case of $S$. aureus, at low concentration $(25 \mu \mathrm{L}) 6 \mathrm{~A}$ exhibits nearly 3 times activity than its parent ligand but high concentration $(100 \mu \mathrm{L})$ only $50 \%$ increase in activity is observed. In case of E.coli, at all concentrations nearly 2 times higher activity is observed for Cu-TCMTSC than its ligand TCMTSC.

\section{CONCLUSION}

From all the above, it is confirmed the synthesis of copper(II) complexes of CCPTSC, HPCPTSC and TCMTSC. All the characterization studies including FT-IR, XRD and thermogravimetric studies confirms the chelation of copper(II) with the thiosemicarbazone ligands. Based on thermogravimetric analysis studies, it is found that complexes of copper are more stable than their chelating agents. XRD studies found change in the morphology of ligands due to the chelation with copper metal ions. Antibacterial studies concludes that the complexes are having higher activity than their parent ligands against all tested bacterial stains. The activity of ligands are enhanced with the chelation of metal ion. The activity of CCPTSC and TCMTSC against E.coli almost doubled due to chelation with copper(II) ions. Cu-CCPTSC complexes shows higher activity against $P$. aeruginosa while other complexes are having with $S$. aureus. To determine the reason for increasing the activity of the free ligands by the 
chelation with copper(II) ions we are planning to establish the structure activity relationship studies.

\section{ACKNOWLEDGEMENT}

One of the authors, N. Rama Jyothi is thankful to Sri Bharathidasan University, Tiruchirapalli for awarding doctor of philosophy for submitting the research work including the present study and also thankful to Khadir Mohideen College, Adhirampattinam for supporting during the research work.

\section{Conflicts of Interest}

The authors declare no conflict of interest.

\section{REFERENCES}

1. Oliveira, C.G.; Canelon I.R.; Silva, M.M.; Coverdale, J.P.C.; Maia, P.I.S.; Batista, A.A.; Castelli, S.; Desideri, A.; Sadler, P.J.; Deflon, V.M. Dalton Trans., 2019, 48, 16509.

2. Adak, P.; Ghosh, B.; Bauza, A.; Frontera, A.; Herron, S.R.; Chattopadhyay, S.K. RSC Adv., 2020, 10, 12735.

3. Kallus, S.; Uhlik, L.; Schoonhoven, S.V.; Pelivan, K.; Berger, W.; Enyedy, E.A.; Hofmann, T.; Heffeter, P.; Kowol, C.R.; Keppler, B.K.; J. Inorg. Biochem., 2019, 190, 85.

4. Yousuf, T.A.; Abu El-Reash, G.M. J. Mol. Struct., 2020, 1201, 127180.

5. Salam, M.A., Alam, M.; Sarker, S.; Rahman, M. M. J. Coord. Chem., 2018, 71, 1593.

6. Khan, S.A.; Asiri, A.M. Steroids., 2017, 124, 23.

7. Srinivasulu, K.; Dhanalakshmi, D.; Anuja, K.; Reddy, K.H. Eur. J. Biomed. Pharm. Sci., 2019, 6, 317.

8. Rama Jyothi, N., Farook., N.A.M.;Vijala Lakshmi, D.; Yadav, P.S. Pharmacophore., 2019, 10, 1.

9. Chan, Y.C.; Ali, A.S. M.; Salleh, B.; Rosli, H.; Quah, C.K. Arabian J. Chem., 2017, 1053493.

10. Ouyang, R.; Yang, Y.; Tong, X.; Yang, Y.; Tao, H.; Zong, T.; Feng, K.; Jia, P.; Cao, P.; Guo, N.; Chang, H.; Zhou, S.; Mial, Y. Inorg. Chem. Commu., 2016, 73, 138.

11. Parsa, F.G.; Mah, F.; Safaralizadeh, R.; Hosseini-Yazdi, S.A.; Mahdavi, M. J. Bio. Inorg. Chem., 2020. https://doi.org/10.1007/ s00775-020-01769-0.

12. Chandra, S.; Vandana, Spectrochim. Acta Part A., 2014, 129, 333.

13. Khan, S.A.; Asiri, A.M.; Amry, K.A.; Malik M.A. The Scientific World Journal., 2014, 2014 Article ID: 592375.

14. Piri, Z.; Shoeli, Z. M.; Assoud, A. Inorg. Chem. Commun., 2017, 84, 122.

15. Bisceglie, F.; Bacci, C.; Vismarra, A.; Barilli, E.; Pioli, M.; Orsoni, N.; Pelosi, G. J. Inorg. Bio Chem., 2020, 203, 110888.

16. Refat, M.S.; El-Deen, I.M.; Anwer, Z.M.; ElGhol, S. J. Mol. Struct., 2009, 920, 149-162.

17. Naveen, R.K.; Tittal, V.D.; Ghule, N.; Kumar, L.; Kumar, K.; Kumar, A. J. Mol. Struct., 2020, 1209, 127951.

28. Kumar, S.; Hansda, A.; Chandra, A.; Kumar, A.; Kumar, M.; Sithambaresan, M.; Faizi, Md. S. H.; Kumar, V.; John, R. P. Polyhedron., 2017, 134, 11-21.

19. Khandani, M.; Sedaghat, T.; Erfani, N.; Haghshenas, M.R.; Khavasi, H.R. J. Mol. Struct., 2013, 1037, 136.

20. Akbari, A.; Ghateazadeh, H.; Takjoo, R.; Nejad, B.S.; Mehrvar, M.; Mague, J.T. J. Mol. Struct., 2019, 1181, 287.

21. Rama Jyothi, N.; Mohamed Farook, N.A. Chem. Sci. Trans., 2018, 7(4), 626.

22. Rama Jyothi, N.; Mohamed Farook, N.A. Chem. Sci. Trans., 2018, 7(4), 634. 